\title{
On the order of a non-abelian representation group of a slim dense near hexagon
}

\author{
Binod Kumar Sahoo • N.S. Narasimha Sastry
}

Received: 2 May 2006 / Accepted: 21 February 2008 / Published online: 4 March 2008

(C) Springer Science+Business Media, LLC 2008

\begin{abstract}
In this paper we study the possible orders of a non-abelian representation group of a slim dense near hexagon. We prove that if the representation group $R$ of a slim dense near hexagon $S$ is non-abelian, then $R$ is a 2-group of exponent 4 and $|R|=2^{\beta}, 1+N P \operatorname{dim}(S) \leq \beta \leq 1+\operatorname{dim} V(S)$, where $N \operatorname{Pdim}(S)$ is the near polygon embedding dimension of $S$ and $\operatorname{dim} V(S)$ is the dimension of the universal representation module $V(S)$ of $S$. Further, if $\beta=1+N P \operatorname{dim}(S)$, then $R$ is necessarily an extraspecial 2-group. In that case, we determine the type of the extraspecial 2-group in each case. We also deduce that the universal representation group of $S$ is a central product of an extraspecial 2-group and an abelian 2-group of exponent at most 4.
\end{abstract}

Keywords Near polygons · Non-abelian representations $\cdot$ Generalized quadrangles $\cdot$ Extraspecial 2-groups

\section{Introduction}

A partial linear space is a pair $S=(P, L)$ consisting of a non-empty 'point-set' $P$ and a 'line-set' $L$ of subsets of $P$ of size at least two such that any two distinct points $x$ and $y$ are contained in at most one line. Such a line, if it exists, is written as $x y$

This work was partially done when B.K. Sahoo was a Research Fellow at the Indian Statistical Institute, Bangalore Center with NBHM fellowship, DAE Grant 39/3/2000-R\&D-II, Govt. of India.

B.K. Sahoo $(\bowtie)$

Department of Mathematics, National Institute of Technology, Rourkela 769008, India

e-mail: binodkumar@gmail.com

N.S.N. Sastry

Statistics and Mathematics Unit, Indian Statistical Institute, 8th Mile, Mysore Road, R.V. College Post, Bangalore 560059, India

e-mail: nnsastry@gmail.com 
and the points $x$ and $y$ are said to be collinear (notation: $x \sim y$ ). If $x$ and $y$ are not collinear, we write $x \nsim y$. If each line of $S$ contains exactly three points, then $S$ is called slim. For $x \in P$ and $A \subseteq P$, we define

$$
x^{\perp}=\{x\} \cup\{y \in P: x \sim y\} \text { and } A^{\perp}=\bigcap_{x \in A} x^{\perp} .
$$

If $P^{\perp}$ is empty, then $S$ is called non-degenerate. A subset of $P$ is a subspace of $S$ if any line containing at least two of its points is contained in it. For a subset $X$ of $P$, the subspace $\langle X\rangle$ generated by $X$ is the intersection of all subspaces of $S$ containing $X$. A geometric hyperplane of $S$ is a subspace of $S$, different from $P$, that meets every line non-trivially. The graph $\Gamma(P)$ with vertex set $P$, two distinct points being adjacent if they are collinear in $S$, is called the collinearity graph of $S$. For $x \in P$ and an integer $i$, we write

$$
\begin{aligned}
\Gamma_{i}(x) & =\{y \in P: d(x, y)=i\}, \\
\Gamma_{\leq i}(x) & =\{y \in P: d(x, y) \leq i\},
\end{aligned}
$$

where $d(x, y)$ denotes the distance between $x$ and $y$ in $\Gamma(P)$. The diameter of $S$ is the diameter of $\Gamma(P)$. If $\Gamma(P)$ is connected, then $S$ is called a connected partial linear space.

\subsection{Representation of a partial linear space}

Let $S=(P, L)$ be a slim partial linear space. If $x, y \in P$ and $x \sim y$, we define $x * y$ by $x y=\{x, y, x * y\}$.

Definition 1.1 ([9], p. 525) A representation of $S$ is a mapping $\psi: x \mapsto\left\langle r_{x}\right\rangle$ from the point set $P$ of $S$ into the set of subgroups of order 2 of a group $R$ such that the following hold:

(i) $R$ is generated by $\operatorname{Im}(\psi)$.

(ii) If $l=\{x, y, x * y\} \in L$, then $\left\{1, r_{x}, r_{y}, r_{x * y}\right\}$ is a Klein four subgroup of $R$.

We write $(R, \psi)$ to mean that $\psi$ is a representation of $S$ with representation group $R$ and say that $(R, \psi)$ is a representation of $S$. We set $R_{\psi}=\left\{r_{x}: x \in P\right\}$. The representation $(R, \psi)$ of $S$ is faithful if $\psi$ is injective, and is abelian or non-abelian according as $R$ is abelian or not. Note that, in [9], 'non-abelian representation' means 'the representation group is not necessarily abelian'.

Let $S$ be a connected slim partial linear space. For an abelian representation of $S$, the representation group can be considered as vector space over $F_{2}$, the field with two elements. If $S$ admits at least one abelian representation, then there exists a unique abelian representation $\rho_{0}$ of $S$ such that any other abelian representation of $S$ is a composition of $\rho_{0}$ and a linear mapping (see [11]). The map $\rho_{0}$ is called the universal abelian representation of $S$. The $F_{2}$ vector space $V(S)$ underlying the universal abelian representation is called the universal representation module of $S$. Considering 
$V(S)$ as an abstract group with the group operation + , it has the presentation

$$
\begin{gathered}
V(S)=\left\langle v_{x}: x \in P ; 2 v_{x}=0 ; v_{x}+v_{y}=v_{y}+v_{x} \text { for } x, y \in P\right. \\
\text { and } \left.v_{x}+v_{y}+v_{x * y}=0 \text { if } x \sim y\right\rangle
\end{gathered}
$$

and $\rho_{0}$ is defined by $\rho_{0}(x)=\left\langle v_{x}\right\rangle$ for $x \in P$.

A representation $\left(R_{1}, \psi_{1}\right)$ of $S$ is a cover of a representation $\left(R_{2}, \psi_{2}\right)$ of $S$ if there exists a group homomorphism $\varphi: R_{1} \rightarrow R_{2}$ such that $\psi_{2}(x)=\varphi\left(\psi_{1}(x)\right)$ for every $x \in P$. If $S$ admits a non-abelian representation, then there is a universal representation $\left(R(S), \psi_{S}\right)$ which is the cover of every other representation of $S$. The universal representation is unique (see [8], p. 306) and the universal representation group $R(S)$ of $S$ has the presentation:

$$
R(S)=\left\langle r_{x}: x \in P, r_{x}^{2}=1, r_{x} r_{y} r_{z}=1 \text { if }\{x, y, z\} \in L\right\rangle .
$$

Whenever we have a representation of $S$, the group spanned by the images of the points is a quotient of $R(S)$. Further,

Lemma $1.2([9]$, p. 525) $V(S)=R(S) /[R(S), R(S)]$.

The general notion of a representation group of a finite partial linear space with $p+1$ points per line for a prime $p$ was introduced by Ivanov [8] in his investigations of Petersen and Tilde geometries (motivated in large measure by questions about the Monster and Baby Monster finite simple groups). A sufficient condition on the partial linear space and on the non-abelian representation of it is given in [12] to ensure that the representation group is a finite $p$-group. For more on non-abelian representations, we refer to [8], also see ([12], Sections 1 and 2). In this paper, we study the possible orders of a non-abelian representation group of a slim dense near hexagon (Theorem 1.6).

\subsection{Near $2 n$-gons}

A near $2 n$-gon is a connected partial linear space $S=(P, L)$ of diameter $n$ such that for each point-line pair $(x, l) \in P \times L, l$ contains a unique point nearest to $x$. Nondegenerate near 4-gons are precisely generalized quadrangles (GQs, for short); that is, non-degenerate partial linear spaces such that for each point-line pair $(x, l)$ with $x \notin l, x$ is collinear with exactly one point of $l$.

Let $S=(P, L)$ be a near $2 n$-gon. The sets $S(x)=\Gamma_{\leq n-1}(x), x \in P$, are special geometric hyperplanes. A subset $C$ of $P$ is convex if every shortest path in $\Gamma(P)$ between two points of $C$ is entirely contained in $C$. A quad is a non-degenerate convex subspace of $P$ of diameter two. Thus a quad carries the structure of a generalized quadrangle. Let $x_{1}, x_{2} \in P$ with $d\left(x_{1}, x_{2}\right)=2$ and $\left|\left\{x_{1}, x_{2}\right\}^{\perp}\right| \geq 2$. If $y_{1}$ and $y_{2}$ are distinct elements of $\left\{x_{1}, x_{2}\right\}^{\perp}$ such that at least one of the lines $x_{i} y_{j}$ contains at least three points, then $x_{1}$ and $x_{2}$ are contained in a unique quad ([13], Proposition 2.5, p. 10). We denote this quad by $Q\left(x_{1}, x_{2}\right)$.

A near $2 n$-gon is called dense if each line contains at least three points and any two distinct points at distance two from each other have at least two common neighbours. 
In a dense near $2 n$-gon, the number of lines through a point is independent of the point ([2], Lemma 19, p. 152). We denote this number by $t+1$. A near $2 n$-gon is said to have parameters $(s, t)$ if each line contains $s+1$ points and each point is contained in $t+1$ lines. A dense near 4-gon with parameters $(s, t)$ is written as an $(s, t)$-GQ.

Theorem 1.3 ([13], Proposition 2.6, p. 12) Let $S=(P, L)$ be a near $2 n$-gon and $Q$ be a quad in $S$. Then, for $x \in P$, either

(i) there is a unique point $y \in Q$ closest to $x$ (depending on $x$ ) and $d(x, z)=$ $d(x, y)+d(y, z)$ for all $z \in Q ;$ or

(ii) the points in $Q$ closest to $x$ form an ovoid $\mathcal{O}_{x}$ of $Q$.

The point-quad pair $(x, Q)$ in Theorem 1.3 is called classical in the first case and ovoidal in the second case. A quad $Q$ in $S$ is classical if $(x, Q)$ is classical for each $x \in P$, otherwise it is ovoidal.

\subsection{Slim dense near hexagons}

A near 6-gon is called a near hexagon. Let $S=(P, L)$ be a slim dense near hexagon. For $x, y \in P$ with $d(x, y)=2$, we write $\left|\Gamma_{1}(x) \cap \Gamma_{1}(y)\right|$ as $t_{2}+1$ (though this depends on $x, y)$. We have $t_{2}<t$. We say that a quad $Q$ in $S$ is of type $\left(2, t_{2}\right)$ if it is a $\left(2, t_{2}\right)-$ GQ. A quad in $S$ is big if it is classical. Thus, if $Q$ is a big quad in $S$, then each point of $S$ has distance at most one from $Q$.

Theorem 1.4 ([1], Theorem 1.1, p. 349) Let $S=(P, L)$ be a slim dense near hexagon. Then $P$ is necessarily finite and $S$ is isomorphic to one of the eleven near hexagons with parameters as given below.

\begin{tabular}{|r|c|c|c|c|c|c|c|c|}
\hline & $|P|$ & $t$ & $t_{2}$ & $\operatorname{dim} V(S)$ & $N P \operatorname{dim}(S)$ & $a_{1}$ & $a_{2}$ & $a_{4}$ \\
\hline$($ i $)$ & 759 & 14 & 2 & 23 & 22 & - & 35 & - \\
\hline$($ ii $)$ & 729 & 11 & 1 & 24 & 24 & 66 & - & - \\
\hline$($ iii $)$ & 891 & 20 & $4^{\star}$ & 22 & 20 & - & - & 21 \\
\hline$($ iv $)$ & 567 & 14 & $2,4^{\star}$ & 21 & 20 & - & 15 & 6 \\
\hline$(v)$ & 405 & 11 & $1,2,4^{\star}$ & 20 & 20 & 9 & 9 & 3 \\
\hline$(v i)$ & 243 & 8 & $1,4^{\star}$ & 18 & 18 & 16 & - & 2 \\
\hline$($ vii $)$ & 81 & 5 & $1,4^{\star}$ & 12 & 12 & 5 & - & 1 \\
\hline$($ viii $)$ & 135 & 6 & $2^{\star}$ & 15 & 8 & - & 7 & - \\
\hline$($ ix $)$ & 105 & 5 & $1,2^{\star}$ & 14 & 8 & 3 & 4 & - \\
\hline$(x)$ & 45 & 3 & $1,2^{\star}$ & 10 & 8 & 3 & 1 & - \\
\hline$(x i)$ & 27 & 2 & $1^{\star}$ & 8 & 8 & 3 & - & - \\
\hline
\end{tabular}

Here, $N P \operatorname{dim}(S)$ is the $F_{2}$-rank of the matrix $A_{3}: P \times P \longrightarrow F_{2}$ defined by $A_{3}(x, y)=1$ if $d(x, y)=3$ and zero otherwise. We add a star if and only if the corresponding quads are big. The number of quads of type $(2, r), r=1,2,4$, containing a given point of $S$ is indicated by $a_{r}$. A '-' in a column means that $a_{r}=0$. 
For a description of the near hexagons $(i)-(i i i)$, see [13] and for $(i v)-(x i)$, see [1]. However, the parameters of these near hexagons suffice for our purposes here. We refer to [5] and [6] for other classification results about slim dense near polygons. For more on near polygons, see [4].

\subsection{Extraspecial 2-groups}

A finite 2-group $G$ is extraspecial if its Frattini subgroup $\Phi(G)$, commutator subgroup $G^{\prime}$ and center $Z(G)$ coincide and have order 2 .

An extraspecial 2-group is of exponent 4 and of order $2^{1+2 m}$ for some integer $m \geq 1$ and the maximum of the orders of its abelian subgroups is $2^{m+1}$ (see [7], Section 20, pp. 78, 79). An extraspecial 2-group $G$ of order $2^{1+2 m}$ is a central product of either $m$ copies of the dihedral group $D_{8}$ of order 8 or $m-1$ copies of $D_{8}$ with a copy of the quaternion group $Q_{8}$ of order 8 . In the former case, $G$ possesses a maximal elementary abelian subgroup of order $2^{1+m}$ and we write $G=2_{+}^{1+2 m}$. If the latter holds, then all maximal abelian subgroups of $G$ are of the type $2^{m-1} \times 4$ and we write $G=2_{-}^{1+2 m}$.

Notation 1.5 For a group $G, G^{*}=G \backslash\{1\}$.

\subsection{The main result}

In this paper, we prove the following.

Theorem 1.6 Let $S=(P, L)$ be a slim dense near hexagon and $(R, \psi)$ be a nonabelian representation of $S$. Then

(i) $R$ is a finite 2-group of exponent 4 and order $2^{\beta}$, where $1+N \operatorname{Pdim}(S) \leq \beta \leq$ $1+\operatorname{dim} V(S)$.

(ii) If $\beta=1+N P \operatorname{dim}(S)$, then $R$ is an extraspecial 2-group. Further, $R=$ $2_{+}^{1+N P \operatorname{dim}(S)}$ except for the near hexagon (vi) in Theorem 1.4. In that case, $R=2_{-}^{1+N P \operatorname{dim}(S)}$.

Section 2 is about some elementary properties of slim dense near hexagons. In Section 3, we study faithful representations of $(2, t)$-GQs. In Section 4, we study non-abelian representations of slim dense near hexagons. We prove Theorem 1.6 in Section 5 .

\section{Elementary properties}

Let $S=(P, L)$ be a slim dense near hexagon. Since a $(2,4)$-GQ admits no ovoids, every quad in $S$ of type $(2,4)$ is big (see Theorem 1.3 ).

Lemma 2.1 ([1], p. 359) Let $Q$ be a quad in $S$ of type (2, $\left.t_{2}\right)$. Then $|P| \geq|Q|(1+$ $\left.2\left(t-t_{2}\right)\right)$. Equality holds if and only if $Q$ is big. In particular, if a quad in $S$ of type $\left(2, t_{2}\right)$ is big then so are all quads in $S$ of that type. 
Let $Q_{1}$ and $Q_{2}$ be two disjoint big quads in $S$. By Lemma 2.1, $Q_{1}$ and $Q_{2}$ are of the same type.

Lemma 2.2 ([1], Proposition 4.3, p. 354) Let $\pi$ be the map from $Q_{1}$ to $Q_{2}$ which takes $x$ to $z_{x}$, where $x \in Q_{1}$ and $z_{x}$ is the unique point in $Q_{2}$ at distance one from $x$. Then

(i) $\pi$ is an isomorphism from $Q_{1}$ to $Q_{2}$.

(ii) The set $Q_{1} * Q_{2}=\left\{x * z_{x}: x \in Q_{1}\right\}$ is a big quad in $S$ disjoint from $Q_{1}$ and $Q_{2}$.

Let $Y$ be the subspace of $S$ generated by $Q_{1}$ and $Q_{2}$. Since $Y$ is the union of $Q_{1}, Q_{2}$ and $Q_{1} * Q_{2}$, it follows that $Y$ is isomorphic to the near hexagon $(x i),(x)$ or (vii) according as $Q_{1}$ and $Q_{2}$ are of type $(2,1),(2,2)$ or $(2,4)$.

Let $\{i, j\}=\{1,2\}$. For $x \in P \backslash Y$, we denote by $x^{j}$ the unique point in $Q_{j}$ at a distance 1 from $x$. For $y \in Q_{i}, z_{y} \in Q_{j}$ is defined as in Lemma 2.2. The following elementary results are useful for us.

Proposition 2.3 For $x \in P \backslash Y, d\left(z_{x^{i}}, x^{j}\right)=1$ and $d\left(z_{x^{1}}, z_{x^{2}}\right)=d\left(x^{1}, x^{2}\right)=2$; that is, $\left\{x^{1}, z_{x^{1}}, x^{2}, z_{x^{2}}\right\}$ is a quadrangle in $\Gamma(P)$.

Proof Since $x \in \Gamma_{1}\left(x^{1}\right) \cap \Gamma_{1}\left(x^{2}\right), d\left(x^{1}, x^{2}\right)=2$. Further, $d\left(x^{i}, x^{j}\right)=d\left(x^{i}, z_{x^{i}}\right)+$ $d\left(z_{x^{i}}, x^{j}\right)$. So $d\left(z_{x^{i}}, x^{j}\right)=1$ and $d\left(z_{x^{1}}, z_{x^{2}}\right)=2$.

Proposition 2.4 Let $l$ be a line of $S$ disjoint from $Y$ and $x, y \in l, x \neq y$. Then, $x^{1} y^{1}=x^{1} z_{x^{2}}$ in $Q_{1}$ if and only if $x^{2} y^{2}=x^{2} z_{x^{1}}$ in $Q_{2}$. In fact, if $x^{1} y^{1}=x^{1} z_{x^{2}}$, then $\left(y^{1}, y^{2}\right)=\left(z_{x^{2}}, x^{2} * z_{x^{1}}\right)$ or $\left(x^{1} * z_{x^{2}}, z_{x^{1}}\right)$.

Proof We have $x^{j} y^{j}=x^{j} z_{x^{i}}$ if and only if $y^{j} \in\left\{z_{x^{i}}, x^{j} * z_{x^{i}}\right\}$. If $y^{j}=x^{j} * z_{x^{i}}$, then $y^{i} \sim x^{i} * z_{x^{j}}$, because $2=d\left(y^{j}, y^{i}\right)=d\left(y^{j}, x^{i} * z_{x^{j}}\right)+d\left(x^{i} * z_{x^{j}}, y^{i}\right)$. Since $y^{i} \sim x^{i}$, it follows that $y^{i}$ is a point in the line $x^{i} z_{x^{j}}$ and so $y^{i}=z_{x^{j}}$.

If $y^{j}=z_{x^{i}}$, then applying the above argument to $(x * y)^{j}=x^{j} * z_{x^{i}}$, we get $(x *$ $y)^{i}=z_{x^{j}}$ and so $y^{i}=x^{i} * z_{x^{j}}$.

Proposition 2.5 Let $l$ be a line of $S$ disjoint from $Y$ and $x, y \in l, x \neq y$. Then $d\left(z_{x^{i}}, z_{y^{j}}\right) \leq 2$ if and only if $x^{i} y^{i}=x^{i} z_{x^{j}}$ in $Q_{i}$.

Proof If $x^{i} y^{i}=x^{i} z_{x^{j}}$ in $Q_{i}$, then $x^{j} y^{j}=x^{j} z_{x^{i}}$ in $Q_{j}$ (Proposition 2.4) and it follows that $d\left(z_{x^{i}}, z_{y^{j}}\right) \leq 2$. Conversely, let $x^{i} y^{i} \neq x^{i} z_{x^{j}}$ in $Q_{i}$. Again by Proposition 2.4, $x^{j} y^{j} \neq x^{j} z_{x^{i}}$ in $Q_{j}$. So $y^{j} \nsim z_{x^{i}}$. Then $d\left(x^{i}, y^{j}\right)=d\left(x^{i}, z_{x^{i}}\right)+$ $d\left(z_{x^{i}}, y^{j}\right)=1+2=3$. This implies that $d\left(z_{x^{i}}, z_{y^{j}}\right)=3$.

Proposition 2.6 Let $Q$ be a big quad in $S$ disjoint from $Y$. For $x, y \in Q$ with $x \nsim y$, $\left\{d\left(z_{x^{1}}, z_{y^{2}}\right), d\left(z_{x^{2}}, z_{y^{1}}\right)\right\}=\{2,3\}$.

Proof By Lemma 2.2, there exists $w \in\{x, y\}^{\perp}$ in $Q$ such that $x^{1} w^{1}=x^{1} z_{x^{2}}$. By Proposition $2.4,\left(w^{1}, w^{2}\right)=\left(z_{x^{2}}, x^{2} * z_{x^{1}}\right)$ or $\left(x^{1} * z_{x^{2}}, z_{x^{1}}\right)$. Assume that $\left(w^{1}, w^{2}\right)=$ 
$\left(z_{x^{2}}, x^{2} * z_{x^{1}}\right)$. Then, $d\left(z_{x^{2}}, z_{y^{1}}\right)=d\left(w^{1}, z_{y^{1}}\right)=d\left(w^{1}, z_{w^{1}}\right)+d\left(z_{w^{1}}, z_{y^{1}}\right)=2$. Now, $y^{2} \sim w^{2}$ and $y^{2} \nsim x^{2}$ in $Q_{2}$ implies that $x^{1} \nsim z_{y^{2}}$. So $d\left(x^{1}, z_{y^{2}}\right)=2$ and $d\left(z_{x^{1}}, z_{y^{2}}\right)=d\left(z_{x^{1}}, x^{1}\right)+d\left(x^{1}, z_{y^{2}}\right)=3$. A similar argument holds if $\left(w^{1}, w^{2}\right)=$ $\left(x^{1} * z_{x^{2}}, z_{x^{1}}\right)$.

\section{Representations of $(2, t)-$ GQs}

Let $S=(P, L)$ be a $(2, t)$-GQ. Then $P$ is finite and $t=1,2$ or 4 . For each value of $t$ there exists a unique generalized quadrangle, up to isomorphism ([3], Theorem 7.3, p. 99). A $k$-arc of $S$ is a set of $k$ pair-wise non-collinear points of $S$. A $k$-arc is complete if it is not contained in a $(k+1)$-arc. A point $x$ is a center of a $k$-arc if $x$ is collinear with every point of it. An ovoid of $S$ is a $k$-arc meeting each line of $S$ non-trivially. A spread of $S$ is a set $K$ of lines of $S$ such that each point of $S$ is in a unique member of $K$. If $O$ (resp., $K$ ) is an ovoid (resp., spread) of $S$, then $|O|=1+2 t$ (resp., $|K|=1+2 t$ ).

Since each line contains three points, each pair of non-collinear points of $S$ is contained in a $(2,1)$-subGQ of $S$. For $t^{\prime}<t$, a $\left(2, t^{\prime}\right)$-subGQ of $S$ and a point outside it generate a $\left(2,2 t^{\prime}\right)$-subGQ in $S$. The minimal number of points which are necessary to generate a $(2, t)$-GQ is equal to 4 if $t=1,5$ if $t=2$ and 6 if $t=4$.

\section{$3.1(2,2)-\mathrm{GQ}$}

Let $S=(P, L)$ be a $(2,2)$-GQ. For any 3 -arc $T$ of $S,\left|T^{\perp}\right|=1$ or 3 . Further, $\left|T^{\perp}\right|=1$ if and only if $T$ is contained in a unique $(2,1)$-subGQ of $S$; and $\left|T^{\perp}\right|=3$ if and only if $T$ is a complete 3 -arc. If $S$ admits a $k$-arc, then $k \leq 5$. Here 5 -arcs are ovoids and $S$ contains six ovoids. Each ovoid is determined by any two of its points. Each point of $S$ is in two ovoids and the intersection of two distinct ovoids is a singleton. Any two non-collinear points of $S$ are in a unique ovoid of $S$ and also in a unique complete 3 -arc of $S$. Any incomplete 3-arc of $S$ is contained in a unique ovoid. Any 4-arc of $S$ is not complete and is contained in a unique ovoid. The intersection of two distinct complete 3-arcs of $S$ is empty or a singleton.

A model for the (2,2)-GQ: Let $\Omega=\{1,2,3,4,5,6\}$. A factor of $\Omega$ is a set of three pair-wise disjoint 2-subsets of $\Omega$. Let $\mathcal{E}$ be the set of all 2-subsets of $\Omega$ and $\mathcal{F}$ be the set of all factors of $\Omega$. Then $|\mathcal{E}|=|\mathcal{F}|=15$ and the pair $(\mathcal{E}, \mathcal{F})$ is a $(2,2)$-GQ.

\section{$3.2(2,4)-G Q$}

Let $S=(P, L)$ be a $(2,4)$-GQ. If $S$ admits a $k$-arc, then $0 \leq k \leq 6$. So $S$ has no ovoids. $S$ admits two disjoint 6-arcs. A 5-arc of $S$ is complete if and only if it is contained in a unique $(2,2)$-subGQ of $S$. Each incomplete 5-arc has exactly one center and each complete 5-arc of $S$ has exactly two centers. Each 4-arc has two centers and is contained in a unique complete 5-arc and in a unique complete 6-arc. Each 3-arc of $S$ has three centers and is contained in a unique $(2,1)$-subGQ of $S$. So any 4-arc of $S$ is contained in a unique $(2,2)$-subGQ of $S$. 
A model for the $(2,4)-\mathbf{Q Q}$ : Let $\Omega, \mathcal{E}$ and $\mathcal{F}$ be as in the model of a $(2,2)-\mathrm{GQ}$. Let $\Omega^{\prime}=\left\{1^{\prime}, 2^{\prime}, 3^{\prime}, 4^{\prime}, 5^{\prime}, 6^{\prime}\right\}$. Take

$$
P=\mathcal{E} \cup \Omega \cup \Omega^{\prime} ; L=\mathcal{F} \cup\left\{\left\{i,\{i, j\}, j^{\prime}\right\}: 1 \leq i \neq j \leq 6\right\}
$$

Then $|P|=27,|L|=45$ and the pair $(P, L)$ is a $(2,4)$-GQ.

\subsection{Representations}

Let $S=(P, L)$ be a $(2, t)-\mathrm{GQ}$ and $(R, \psi)$ be a representation of $S$.

Proposition 3.1 $R$ is an elementary abelian 2-group.

Proof Let $x, y \in P$ and $x \nsim y$. Let $T$ be a $(2,1)$-subGQ of $S$ containing $x$ and $y$. Let $\{x, y\}^{\perp} \cap T=\{a, b\}$. Then $\left[r_{x}, r_{y}\right]=1$, because $r_{b} r_{y}=r_{y} r_{b}, r_{b} r_{x}=r_{x} r_{b}$ and $r_{(a * x) *(b * y)}=r_{(a * y) *(b * x)}$. So $R$ is abelian.

For the rest of this section we assume that $\psi$ is faithful.

Proposition 3.2 The following hold:

(i) $|R|=2^{4}$ if $t=1$;

(ii) $|R|=2^{4}$ or $2^{5}$ if $t=2$, and both possibilities occur;

(iii) $|R|=2^{6}$ if $t=4$.

Proof Since $S$ is generated by a set of $k$ points where $(t, k) \in\{(1,4),(2,5),(4,6)\}$, $F_{2}$-dimension of $R$ is at most $k$. So $|R| \leq 2^{k}$.

(i) If $t=1$, then $|R| \geq 2^{4}$ because $|P|=9$ and $\psi$ is faithful. So $|R|=2^{4}$.

(ii) If $t=2$, then $|R| \geq 2^{4}$ because $S$ contains a (2,1)-subGQ. The rest follows from the fact that $S$ has a symplectic embedding in an $F_{2}$-vector space of dimension 4 as well as an orthogonal embedding in an $F_{2}$-vector space of dimension 5.

(iii) We prove this after Proposition 3.3.

The following is a partial converse to the fact that $r_{x} r_{y} \in R_{\psi}$ for $x, y \in P$ with $x \sim y$. Recall that $R_{\psi}=\left\{r_{x}: x \in P\right\}$.

Proposition 3.3 Assume that $(t,|R|) \neq\left(2,2^{4}\right)$. If $r_{x} r_{y} \in R_{\psi}$ for distinct $x, y \in P$, then $x \sim y$.

Proof Let $z \in P$ be such that $r_{z}=r_{x} r_{y}$. If $x \nsim y$, then $T=\{x, y, z\}$ is a 3-arc of $S$ because $\psi$ is faithful. There is no (2,1)-subGQ of $S$ containing $T$ because the subgroup of $R$ generated by the image of such a subGQ is of order $2^{4}$ (Proposition 3.2(i)). Every 3-arc of a $(2,4)$-GQ is contained in a unique $(2,1)$-subGQ. So $t=2$ and $T$ is a complete 3 -arc. Let $Q$ be a $(2,1)$-subGQ of $S$ containing $x$ and $y$. Then $z \notin Q$ and $P=\langle Q, z\rangle$. Since $r_{z} \in\langle\psi(Q)\rangle,|R|=|\langle\psi(Q)\rangle|=2^{4}$, a contradiction to the assumption. 
If $(t,|R|)=\left(2,2^{4}\right)$, then Proposition 3.3 is not true because in this case $R^{*}=R_{\psi}$, so $r_{x} r_{y} \in R_{\psi}$ for non-collinear points $x$ and $y$.

Proof of Proposition 3.2(iii) If $t=4$, then there are 16 points of $S$ not collinear with a given point $x$. By Proposition 3.3, $\left|R^{*} \backslash R_{\psi}\right| \geq 16$. Thus, $|R|>2^{5}$ and so $|R|=2^{6}$. This completes the proof.

Corollary 3.4 Let $t=4$ and $Q$ be $a(2,2)-s u b G Q$ of $S$. Then $|\langle\psi(Q)\rangle|=2^{5}$.

Proof This follows from Proposition 3.2(iii) and the fact that $P=\langle Q, x\rangle$ for $x \in$ $P \backslash Q$.

Proposition 3.5 If $t=2$, then $|R|=2^{4}$ if and only if $r_{a} r_{b} r_{c}=1$ for every complete 3-arc $\{a, b, c\}$ of $S$.

Proof Let $T=\{a, b, c\}$ be a complete 3 -arc of $S$ and $Q$ be a (2,1)-subGQ of $S$ containing $a$ and $b$. Then $c \notin Q$ and $P=\langle Q, c\rangle$.

If $r_{a} r_{b} r_{c}=1$, then $r_{c} \in\langle\psi(Q)\rangle$ and $|R|=|\langle\psi(Q)\rangle|=2^{4}$. Now, assume that $|R|=$ $2^{4}$. Let $\{x, y\}=\{a, b\}^{\perp} \cap Q$. Then $x, y \in T^{\perp}$, since $T$ is a complete 3 -arc. Let $z$ be the point in $Q$ such that $\{x, y, z\}$ is a 3-arc in $Q$. Then $c \sim z$ and $r_{z}=\left(r_{a} r_{x}\right)\left(r_{b} r_{y}\right)$. Since $H=\left\langle r_{y}: y \in x^{\perp}\right\rangle$ is a maximal subgroup of $R$ ([10], 4.2.4, p. 68), $|H|=2^{3}$. So $r_{c}=r_{a} r_{b}$ or $r_{a} r_{b} r_{x}$, since $\psi$ is faithful. If the latter holds then $r_{c * z}=r_{y}$, which is not possible because $\psi$ is faithful and $y \neq c * z$. Hence $r_{c}=r_{a} r_{b}$.

Corollary 3.6 Assume that $(t,|R|)=\left(2,2^{4}\right)$. Let $T=\{a, b, c\} \subset P$ be such that $r_{a} r_{b} r_{c}=1$. Then $T$ is a line or a complete 3-arc.

Proof Assume that $T$ is not a line. Then, since $\psi$ is faithful, $T$ is a 3 -arc. We show that $T$ is complete. Suppose that $T$ is not complete. Let $\{a, b, d\}$ be the complete 3arc of $S$ containing $a$ and $b$. Then $r_{a} r_{b} r_{d}=1$ (Proposition 3.5) and $c \neq d$. So $r_{c}=r_{d}$, contradicting the fact that $\psi$ is faithful.

Lemma 3.7 If $S$ contains a 3-arc $T=\{a, b, c\}$ such that $r_{a} r_{b} r_{c} \in R_{\psi}$, then $(t,|R|)=$ $\left(2,2^{4}\right)$. In particular, $T$ is incomplete.

Proof Let $x \in P$ be such that $r_{x}=r_{a} r_{b} r_{c}$. Since $\psi$ is faithful, $x \notin T$. Let $t=2$. If $T$ is complete, then $|R|=2^{5}$ (Proposition 3.5) and $x$ is collinear with at least one point of $T$, say $x \sim a$. Then $r_{b} r_{c}=r_{x} r_{a}=r_{x * a} \in R_{\psi}$, a contradiction to Proposition 3.3. Thus, $T$ is incomplete if $t=2$.

Let $Q_{1}$ be the unique (2,1)-subGQ of $S$ containing $T$. If $x \in Q_{1}$, then $\left\langle\psi\left(Q_{1}\right)\right\rangle=$ $\left\langle r_{a}, r_{b}, r_{c}, r_{x}\right\rangle$ would be of order $2^{3}$, contradicting Proposition 3.2(i). So $x \notin Q_{1}$ and $t \neq 1$. Let $Q_{2}$ be the (2,2)-subGQ of $S$ generated by $Q_{1}$ and $x$. Then $\left|\left\langle\psi\left(Q_{2}\right)\right\rangle\right|=2^{4}$, and so $t \neq 4$ (Corollary 3.4). Thus $t=2$ and $|R|=\left|\left\langle\psi\left(Q_{2}\right)\right\rangle\right|=2^{4}$.

Lemma 3.8 Let $a, b \in P$ with $a \nsim b$. Set $A=\left\{r_{a} r_{x}: x \nsim a\right\}$ and $B=\left\{r_{b} r_{x}: x \nsim b\right\}$. Then $|A \cap B|=t+2$. 
Proof It is enough to prove that $r_{a} r_{x}=r_{b} r_{y}$ for $r_{a} r_{x} \in A, r_{b} r_{y} \in B$ if and only if either $x=b$ and $y=a$ holds or there exists a point $c$ such that $\{c, a, y\}$ and $\{c, b, x\}$ are lines. We need to prove the 'only if' part. Since $\psi$ is faithful, $x \neq b$ if and only if $y \neq a$. Assume that $x \neq b$ and $y \neq a$. For this, we show that $y \sim a$ and $x \sim b$. Then $r_{a * y}=r_{a} r_{y}=r_{b} r_{x}=r_{b * x}$. Since $\psi$ is faithful, it would then follow that $a * y=b * x$ and this would be our choice of $c$.

First, assume that $(t,|R|) \neq\left(2,2^{4}\right)$. Since $a \nsim b, r_{a} r_{b} \notin R_{\psi}$ by Proposition 3.3. Since $r_{x} r_{y}=r_{a} r_{b}$, Proposition 3.3 again implies that $x \nsim y$. Now, $r_{a} r_{b} r_{y}=r_{x} \in R_{\psi}$. By Lemma 3.7, $\{a, b, y\}$ is not a 3-arc. This implies that $y \sim a$. By a similar argument, $x \sim b$.

Now, assume that $(t,|R|)=\left(2,2^{4}\right)$. Suppose that $x \nsim b$. Then $T=\{a, b, x\}$ is a 3-arc of $S$. By Proposition 3.7, $T$ is incomplete. Let $Q$ be the (2,1)-subGQ in $S$ containing $T$ and let $\{c, d\}=\{a, b\}^{\perp} \cap Q$. Then $r_{x}=r_{a} r_{b} r_{c} r_{d}=r_{x} r_{y} r_{c} r_{d}$. So $r_{y} r_{c} r_{d}=1$. By Corollary 3.6, $\{c, d, y\}$ is a complete 3 -arc. Since $b \in\{c, d\}^{\perp}$, it follows that $b \in\{c, d, y\}^{\perp}$, a contradiction to that $b \nsim y$. So $x \sim b$. A similar argument shows that $y \sim a$.

Proposition 3.9 Let $K=R^{*} \backslash R_{\psi}$. Each element of $K$ is of the form $r_{y} r_{z}$ for some $y \nsim z$ in $P$, except when $(t,|R|)=\left(2,2^{5}\right)$. In this case, exactly one element, say $\alpha$, of $K$ can't be expressed in this way. Moreover, $\alpha=r_{u} r_{v} r_{w}$ for every complete 3-arc $\{u, v, w\}$ of $S$.

Proof Since $K$ is empty when $(t,|R|)=\left(2,2^{4}\right)$, we assume that $(t,|R|)=\left(1,2^{4}\right)$, $\left(2,2^{5}\right)$ or $\left(4,2^{6}\right)$. Fix $a, b \in P$ with $a \nsim b$. Then $r_{a} r_{b} \in K$ (Proposition 3.3). Let $A$ and $B$ be as in Lemma 3.8, and set

$$
C=\left\{r_{a} r_{b} r_{x}:\{a, b, x\} \text { is a } 3 \text {-arc which is incomplete if } t=2\right\} .
$$

By Proposition 3.3, $A \subseteq K$ and $B \subseteq K$ and by Lemma 3.7, $C \subseteq K$. Each element of $C$ corresponds to a 3 -arc which is contained in a (2,1)-subGQ of $S$. Let $r_{a} r_{b} r_{x} \in$ $C$ and $Q$ be the (2,1)-subGQ of $S$ containing the 3 -arc $\{a, b, x\}$. If $\{a, b\}^{\perp} \cap Q=$ $\{p, q\}$, then $r_{a * p} r_{b * q}=r_{x}$ implies that $r_{a} r_{b} r_{x}=r_{p} r_{q}$. Thus, every element of $C$ can be expressed in the required form.

By Proposition 3.3, $A \cap C$ and $B \cap C$ are empty. By Lemma 3.8, $|A \cap B|=t+2$. Then an easy count shows that

$$
|A \cup B \cup C|=\left\{\begin{array}{ll}
10 t-4 & \text { if } t=1 \text { or } 4 \\
10 t-5 & \text { if } t=2
\end{array} .\right.
$$

So $K=A \cup B \cup C$ if $t=1$ or 4 , and $K \backslash(A \cup B \cup C)$ is a singleton if $t=2$. This proves the proposition for $t=1,4$ and tells that if $(t,|R|)=\left(2,2^{5}\right)$, then at most one element of $K$ can't be written in the desired form.

Now, let $(t,|R|)=\left(2,2^{5}\right)$ and $T=\{u, v, w\}$ be a complete 3 -arc of $S$. By Lemma 3.7, $\alpha=r_{u} r_{v} r_{w} \in K$. Suppose that $\alpha=r_{x} r_{y}$ for some $x, y \in P$. Then $x \nsim y$ by Lemma 3.7 and $\{x, y\} \cap T=\Phi$ by Proposition 3.3. Suppose that $x \in T^{\perp}$ and $Q$ be the $(2,1)$-subGQ of $S$ generated by $\{x, u, v, y\}$. Since $w \notin Q$ and $r_{w}=r_{u} r_{v} r_{x} r_{y}$, it follows that $|R|=2^{4}$, a contradiction. So, $x \notin T^{\perp}$. Similarly, $y \notin T^{\perp}$. Thus, each 
of $x$ and $y$ is collinear with exactly one point of $T$. Let $x \sim u$. Then $y \nsim x * u$, since $x * u \in T^{\perp}$ and $\alpha=r_{x} r_{y}$. Let $U$ be the (2,1)-subGQ of $S$ generated by $\{u, x, y, v\}$. Note that $y \sim u$ in $U$. Let $z$ be the unique point in $U$ such that $\{u, v, z\}$ is a 3 -arc of $U$. Then $r_{z}=r_{x} r_{y} r_{u} r_{v}=r_{w}$. Since $w \neq z$ (in fact, $w \notin U$ ), this is a contradiction to the faithfulness of $\psi$. Thus, $\alpha$ can't be expressed as $r_{x} r_{y}$ for any $x, y$ in $P$. This, together with the last sentence of the previous paragraph, implies that $\alpha$ is independent of the complete 3-arc $T$ of $S$.

\section{Initial results}

Let $S=(P, L)$ be a slim dense near hexagon and $(R, \psi)$ be a non-abelian representation of $S$. For $x \in P$ and $y \in \Gamma_{\leq 2}(x),\left[r_{x}, r_{y}\right]=1$ : if $d(x, y)=2$, we apply Proposition 3.1 to the restriction of $\psi$ to the quad $Q(x, y)$. From ([12], Theorem 2.9, see Example 2.2 of [12]) applied to $S$, we have

Proposition 4.1 The following hold:

(i) For $x, y \in P,\left[r_{x}, r_{y}\right] \neq 1$ if and only if $d(x, y)=3$. In that case, $\left\langle r_{x}, r_{y}\right\rangle$ is a dihedral group $2_{+}^{1+2}$ of order 8 .

(ii) $R$ is a finite 2-group of exponent $4,\left|R^{\prime}\right|=2$ and $R^{\prime}=\Phi(R) \subseteq Z(R)$.

(iii) $r_{x} \notin Z(R)$ for each $x \in P$ and $\psi$ is faithful.

We write $R^{\prime}=\langle\theta\rangle$ throughout. Since $R^{\prime}$ is of order two, Lemma 1.2 implies

Corollary $4.2|R| \leq 2^{1+\operatorname{dim} V(S)}$.

Proposition 4.3 $R$ is a central product $E \circ Z(R)$ of an extraspecial 2-subgroup $E$ of $R$ and $Z(R)$.

Proof We consider $V=R / R^{\prime}$ as a vector space over $F_{2}$. The map $f: V \times V \longrightarrow F_{2}$, taking $(x Z, y Z)$ to 0 or 1 accordingly $[x, y]=1$ or not, is a symplectic bilinear form on $V$. This form is non-degenerate if and only if $R^{\prime}=Z(R)$. Let $W$ be a complement in $V$ of the radical of $f$ and $E$ be its inverse image in $R$. Then $E$ is extraspecial and the proposition follows.

From Proposition 4.3 it follows that the universal representation group of $S$ is a central product of an extraspecial 2-group and an abelian 2-group of exponent at most 4.

Corollary 4.4 Let $M$ be an abelian subgroup of $R$ of order $2^{m}$ intersecting $Z(R)$ trivially. Then $|R| \geq 2^{2 m+1}$. Equality holds if and only if $R$ is extraspecial and $M$ is a maximal abelian subgroup of $R$ intersecting $Z(R)$ trivially.

The following lemma is useful for us.

Lemma 4.5 Let $x \in P$ and $Y \subseteq \Gamma_{3}(x)$. Then $\left[r_{x}, \prod_{y \in Y} r_{y}\right]=1$ if and only if $|Y|$ is even. 
Proof Since $R^{\prime} \subseteq Z(R),\left[r_{x}, \prod_{y \in Y} r_{y}\right]$ is well-defined (though $\prod_{y \in Y} r_{y}$ depends on the order of multiplication). Let $y, z \in \Gamma_{3}(x)$ be distinct. The subgraph of $\Gamma(P)$ induced on $\Gamma_{3}(x)$ is connected (see [2], Corollary to Theorem 3, p. 156). Let $y=y_{0}$, $y_{1}, \ldots, y_{k}=z$ be a path in $\Gamma_{3}(x)$. Then $r_{y} r_{z}=\Pi r_{y_{i} * y_{i+1}}(0 \leq i \leq k-1)$. Since $d\left(x, y_{i} * y_{i+1}\right)=2,\left[r_{x}, r_{y} r_{z}\right]=1$. Now, the result follows from Theorem 4.1 $(i)$.

Notation 4.6 For a quad $Q$ in $S$, we denote by $M_{Q}$ the elementary abelian 2-subgroup of $R$ generated by $\psi(Q)$.

Proposition 4.7 Let $Q$ be a quad in $S$ and $M_{Q} \cap Z(R) \neq\{1\}$. Then $Q$ is of type $(2,2),\left|M_{Q}\right|=2^{5}$ and $M_{Q} \cap Z(R)=\left\{1, r_{a} r_{b} r_{c}\right\}$ for every complete 3-arc $\{a, b, c\}$ of $S$.

Proof Suppose that $M_{Q} \cap Z(R) \neq\{1\}$ and $1 \neq m \in M_{Q} \cap Z(R)$. Then $m \neq r_{x}$ for each $x \in P$ (Proposition 4.1(iii)). If $Q$ is of type $(2,1)$ or $(2,4)$, then by Proposition 3.9, $m=r_{y} r_{z}$ for some $y, z \in Q, y \nsim z$. Choose $w \in P \backslash Q$ with $w \sim y$. Then $\left[r_{w}, r_{z}\right]=\left[r_{w}, r_{y} r_{z}\right]=\left[r_{w}, m\right]=1$. But $d(w, z)=3$ by Theorem $1.3(i)$, a contradiction to Proposition 4.1(i).

So $Q$ is a (2,2)-GQ. If $\left|M_{Q}\right|=2^{4}$, then $M_{Q}^{*}=\left\{r_{x}: x \in Q\right\}$ and $m=r_{x} \in Z(R)$ for some $x \in Q$, contradicting Proposition 4.1(iii). So $\left|M_{Q}\right|=2^{5}$. Now, either $m=$ $r_{u} r_{v}$ for some $u, v \in Q, u \nsim v$ or $m=r_{a} r_{b} r_{c}$ for every complete 3-arc $\{a, b, c\}$ of $Q$ (Proposition 3.9). The above argument again implies that the first possibility does not occur.

Proposition 4.8 Let $Q$ and $Q^{\prime}$ be two disjoint big quads in $S$ of type $\left(2, t_{2}\right), t_{2} \neq 2$. Then $M_{Q} \cap M_{Q^{\prime}}=\{1\}$.

Proof Suppose that $M_{Q} \cap M_{Q^{\prime}} \neq\{1\}$ and $1 \neq m \in M_{Q} \cap M_{Q^{\prime}}$. Assume that $m=r_{x}$ for some $x \in Q$. Choose a point $w \in Q^{\prime}$ with $d(x, w)=3$. Then $\left[r_{x}, r_{w}\right]=\left[m, r_{w}\right]=$ 1 , since $M_{Q^{\prime}}$ is abelian. This contradicts Proposition 4.1(i).

So, $m \neq r_{x}$ for each $x \in P$. Since $Q$ is of type $(2,1)$ or $(2,4), m=r_{y} r_{z}$ for some $y, z \in Q$ with $y \nsim z$ (Proposition 3.9). Choose $w \in Q^{\prime}$ with $w \sim y$. This is possible since $Q^{\prime}$ is big. Then $d(w, z)=3$ and $\left[r_{w}, r_{z}\right]=\left[r_{w}, r_{y} r_{z}\right]=\left[r_{w}, m\right]=1$, again a contradiction to Proposition 4.1(i).

Proposition 4.9 Let $Q$ be a quad in $S$ of type $(2,2)$. Then $Q$ is ovoidal if and only if $\left|M_{Q}\right|=2^{5}$ and $M_{Q} \cap Z(R)=\{1\}$.

Proof First, assume that $Q$ is ovoidal and let $z \in P \backslash Q$ be such that the pair $(z, Q)$ is ovoidal. Let $\mathcal{O}_{z}=\left\{x_{1}, \cdots, x_{5}\right\}$ be the ovoid of $Q$ defined as in Theorem 1.3(ii). If $\left|M_{Q}\right|=2^{4}$, then for the complete 3 -arc $\left\{x_{1}, x_{2}, y\right\}$ of $Q$ containing $x_{1}$ and $x_{2}$, $d(y, z)=3$ and $r_{x_{1}} r_{x_{2}} r_{y}=1$ (Proposition 3.5). But $\left[r_{z}, r_{y}\right]=\left[r_{z}, r_{x_{1}} r_{x_{2}} r_{y}\right]=1$, a contradiction to Proposition 4.1 $(i)$. So $\left|M_{Q}\right|=2^{5}$. Suppose that $M_{Q} \cap Z(R) \neq\{1\}$ and $1 \neq m \in M_{Q} \cap Z(R)$. By Proposition 4.7, $m=r_{a} r_{b} r_{c}$ for every complete 3-arc $\{a, b, c\}$ of $Q$. In particular, for the complete 3 -arc $\left\{x_{1}, x_{2}, y\right\}$ of $Q$ containing $x_{1}$ and $x_{2}$, the above argument leads to a contradiction. So $M_{Q} \cap Z(R)=\{1\}$. 
Now, assume that $\left|M_{Q}\right|=2^{5}$ and $M_{Q} \cap Z(R)=\{1\}$. Suppose that $Q$ is classical and let $\{a, b, c\}$ be a complete 3 -arc of $Q$. Then, by Proposition 3.5, $r_{a} r_{b} r_{c} \neq 1$. Since $(x, Q)$ is classical for each $x \in P \backslash Q$, either each of $a, b, c$ is at a distance two from $x$ or exactly two of them are at a distance three from $x$. In either case, $\left[r_{x}, r_{a} r_{b} r_{c}\right]=1$ (see Lemma 4.5). So $1 \neq r_{a} r_{b} r_{c} \in M_{Q} \cap Z(R)$, a contradiction.

\section{Proof of Theorem 1.6}

Let $S=(P, L)$ be a slim dense near hexagon and let $(R, \psi)$ be a non-abelian representation of $S$. By Proposition 4.1(ii), $R$ is a finite 2-group of exponent 4. By Corollary $4.2,|R| \leq 2^{1+\operatorname{dim} V(S)}$. For each of the near hexagons in Theorem 1.4, except (vi), we find an elementary abelian subgroup of $R$ of order $2^{\xi}, 2 \xi=N \operatorname{Pdim}(S)$, intersecting $Z(R)$ trivially. Then by Corollary $4.4,|R| \geq 2^{1+2 \xi}$ and $R=2_{+}^{1+2 \xi}$ if equality holds. For the near hexagon $(v i)$ we prove in Subsection 5.3 that $R=2_{-}^{1+2 \xi}$, thus completing the proof of Theorem 1.6.

\subsection{The near hexagons (vii) to $(x i)$}

Let $S=(P, L)$ be one of the near hexagons (vii) to $(x i)$ and $Q$ be a big quad in $S$. Set $M=M_{Q}$. Then, by Proposition 4.7, $M \cap Z(R)=\{1\}$ and $|M|=2^{4}$ or $2^{6}$ according as $Q$ is of type $(2,1)$ or $(2,4)$. If $Q$ is of type $(2,2)$, then $|M|=2^{4}$ or $2^{5}$. Also, if $|M|=2^{5}$, then $|M \cap Z(R)|=2$ because $Q$ is classical (Propositions 4.7 and 4.9). Thus, $R$ has an elementary abelian subgroup of order $2^{\xi}$ intersecting $Z(R)$ trivially.

\subsection{The near hexagons $(i)$ to $(v)$}

Let $S=(P, L)$ be one of the near hexagons $(i)$ to $(v)$. Fix $a \in P$ and $b \in \Gamma_{3}(a)$. Let $l_{1}, \cdots, l_{t+1}$ be the lines containing $a, x_{i}$ be the point in $l_{i}$ with $d\left(b, x_{i}\right)=2$ and $A=\left\{x_{i}: 1 \leq i \leq t+1\right\}$. For a subset $X$ of $A$, we set $T_{X}=\left\{r_{x}: x \in X\right\}, M_{X}=\left\langle T_{X}\right\rangle$ and $M=\left\langle r_{b}\right\rangle M_{X}$. Then $M_{X}$ and $M$ are elementary abelian 2-subgroups of $R$.

\section{Proposition 5.1 Let $X$ be a subset of A such that}

(i) $M_{X} \cap Z(R)=\{1\}$,

(ii) $T_{X}$ is linearly independent.

Then, $|M|=2^{|X|+1}$ and $M \cap Z(R)=\{1\}$. In particular, $|R| \geq 2^{2|X|+3}$.

Proof By (ii), $2^{|X|} \leq|M| \leq 2^{|X|+1}$. If $|M|=2^{|X|}$, then $r_{b}$ can be expressed as a product of some of the elements $r_{x}, x \in X$. Since $\left[r_{a}, r_{x}\right]=1$ for $x \in X$, it follows that $\left[r_{a}, r_{b}\right]=1$, a contradiction to Proposition 4.1(i). So $|M|=2^{|X|+1}$. Suppose that $M \cap Z(R) \neq\{1\}$ and $1 \neq z \in M \cap Z(R)$. Let $z=\prod_{y \in X \cup\{b\}} r_{y}^{i_{y}}, i_{y} \in\{0,1\}$. Since $\left[r_{x}, z\right]=1, i_{b}=0$ by the above argument. It follows that $z \in M_{X}$, a contradiction to (i). So $M \cap Z(R)=\{1\}$. 
By Corollary $4.4,|R| \geq 2^{2(|X|+1)+1}=2^{2|X|+3}$.

A subset $X$ of $A$ is good if ( $i$ ) and (ii) of Proposition 5.1 hold. In the rest of this Section, we find good subsets of $A$ of size $(\xi-1)$, thus completing the proof of Theorem 1.6 for the near hexagons $(i)$ to $(v)$. The next Lemma gives a necessary condition for a subset of $A$ to be good.

Lemma 5.2 Let $X$ be a subset of $A$ which is not good, $\alpha \in M_{X} \cap Z(R)$ (possibly $\alpha=1)$ and

$$
\alpha=\prod_{x_{k} \in X} r_{x_{k}}^{i_{k}}
$$

where $i_{k} \in\{0,1\}$. Set $B=\left\{k: x_{k} \in X\right\}, B^{\prime}=\left\{k \in B: i_{k}=1\right\}$ and $A_{i, j}=\left\{k \in B^{\prime}\right.$ : $\left.x_{k} \in Q\left(x_{i}, x_{j}\right)\right\}$ for $1 \leq i \neq j \leq t+1$. Assume that $B^{\prime}$ is non-empty when $\alpha=1$. Then

(i) $\left|B^{\prime}\right| \geq 3$,

(ii) $\left|B^{\prime}\right|$ is even if and only if $\left|A_{i, j}\right|$ is even.

Proof (i) $\left|B^{\prime}\right| \geq 2$ because $r_{x_{k}} \notin Z(R)$ for each $k$ (Proposition 4.1(iii)). If $\left|B^{\prime}\right|=2$, then $r_{x} r_{y}=\alpha$ for some pair of distinct $x, y \in X$. Since $\psi$ is faithful and $r_{x}, r_{y}$ are involutions, $\alpha \neq 1$. For the quad $Q=Q(x, y), 1 \neq \alpha \in M_{Q} \cap Z(R)$. By Proposition 4.7, $Q$ is a $(2,2)-\mathrm{GQ}$ and $r_{a} r_{b} r_{c}=\alpha$ for each complete 3 -arc $\{a, b, c\}$ of $Q$. In particular, if $\{x, y, w\}$ is the complete 3 -arc of $Q$ containing $x$ and $y$, then $r_{x} r_{y} r_{w}=\alpha$. It follows that $r_{w}=1$, a contradiction. So $\left|B^{\prime}\right| \geq 3$.

(ii) Let $w \in Q\left(x_{i}, x_{j}\right)$ and $w \nsim a$. For each $m \in B_{i, j}^{\prime}=B^{\prime} \backslash A_{i, j}, x_{m} \sim a$ and $x_{m} \notin Q\left(x_{i}, x_{j}\right)$. So $d\left(w, x_{m}\right)=3$. Now, $\left[r_{w}, \prod_{m \in B_{i, j}^{\prime}} r_{x_{m}}\right]=\left[r_{w}, \prod_{m \in B^{\prime}} r_{x_{m}}\right]=\left[r_{w}, \alpha\right]=$ 1 , since $\alpha \in Z(R)$. So $\left|B_{i, j}^{\prime}\right|$ is even by Lemma 4.5. This implies (ii).

In what follows, for any subset $X$ of $A$ which is not good, $B^{\prime}$ is defined relative to an expression as in (1) for an arbitrary but fixed element of $M_{X} \cap Z(R)$. Any quad $Q$ in $S$ containing the point $a$ is determined by any two distinct points $x_{i}$ and $x_{j}$ of $A$ that are contained in $Q$. In that case we sometimes denote by $A_{Q}$ the set $A_{i, j}$ defined in Lemma 5.2.

\subsubsection{The near hexagon $(i)$}

There are 7 quads in $S$ containing the point $x_{1} \in A$. This partitions the 14 points $\left(\neq x_{1}\right)$ of $A$, say

$$
\left\{x_{2}, x_{3}\right\} \cup\left\{x_{4}, x_{5}\right\} \cup\left\{x_{6}, x_{7}\right\} \cup\left\{x_{8}, x_{9}\right\} \cup\left\{x_{10}, x_{11}\right\} \cup\left\{x_{12}, x_{13}\right\} \cup\left\{x_{14}, x_{15}\right\} .
$$

Consider the quad $Q\left(x_{10}, x_{12}\right)$. We may assume that $Q\left(x_{10}, x_{12}\right) \cap A=\left\{x_{10}, x_{12}, x_{15}\right\}$. Let $X=\left\{x_{2}, x_{3}, x_{4}, x_{5}, x_{6}, x_{7}, x_{8}, x_{10}, x_{12}, x_{14}\right\}$. Then $|X|=10$. We show that $X$ is a good subset of $A$.

Assume otherwise. Let $C_{1}=\{8,10,12,14\}$ and $C_{2}=B \backslash C_{1}$. For $k \in C_{1}$, $Q\left(x_{1}, x_{k}\right) \cap A=\left\{x_{1}, x_{k}, x_{k+1}\right\}$. So $A_{1, k} \subseteq\{k\}$. By Lemma 5.2(ii), either $C_{1} \subseteq B^{\prime}$ 
or $C_{1} \cap B^{\prime}$ is empty. If $C_{1} \subseteq B^{\prime}$, then $A_{1,14}=\{14\}, A_{10,12}=\{10,12\}$ and by Lemma 5.2(ii), $\left|B^{\prime}\right|$ would be both odd and even.

So $C_{1} \cap B^{\prime}$ is empty. Then $B^{\prime} \subseteq C_{2}$. Since $A_{1,8}$ is empty, $\left|B^{\prime}\right|$ is even. Choose $j \in B^{\prime}$ (see Lemma 5.2(i)). Observe that there exists a $k \in\{8, \cdots, 15\}$ such that $Q\left(x_{j}, x_{k}\right) \cap\left\{x_{i}: i \in C_{2}\right\}=\left\{x_{j}\right\}$. Then $A_{j, k}=\{j\}$ and $\left|B^{\prime}\right|$ is odd also, a contradiction.

\subsubsection{The near hexagon (ii)}

Let $X=\left\{x_{i}: 1 \leq i \leq 11\right\}$. Then $|X|=11$. Also $X$ is a good subset of $A$. Otherwise, for some $i, j \in B^{\prime}$ with $i \neq j$ (see Lemma 5.2(i)), $A_{i, j}=\{i, j\}$ and $A_{i, 12}=\{i\}$ and, by Lemma 5.2(ii), $\left|B^{\prime}\right|$ would be both even and odd.

\subsubsection{The near hexagon (iii)}

Let $Q_{1}, \cdots, Q_{5}$ be the five (big) quads in $S$ containing $x_{1}$ and $a$. Let

$$
\begin{aligned}
& Q_{1} \cap A=\left\{x_{1}, x_{2}, x_{3}, x_{4}, x_{5}\right\}, \\
& Q_{2} \cap A=\left\{x_{1}, x_{6}, x_{7}, x_{8}, x_{9}\right\}, \\
& Q_{3} \cap A=\left\{x_{1}, x_{10}, x_{11}, x_{12}, x_{13}\right\}, \\
& Q_{4} \cap A=\left\{x_{1}, x_{14}, x_{15}, x_{16}, x_{17}\right\}, \\
& Q_{5} \cap A=\left\{x_{1}, x_{18}, x_{19}, x_{20}, x_{21}\right\} .
\end{aligned}
$$

Let $X=\left\{x_{2}, x_{3}, x_{4}, x_{5}, x_{6}, x_{7}, x_{8}, x_{10}, x_{14}\right\}$. Then $|X|=9$. We show that $X$ is a good subset of $A$. Assume otherwise. Since $Q_{5} \cap X$ is empty, $A_{Q_{5}}$ is empty and, by Lemma 5.2(ii), $\left|B^{\prime}\right|$ and $\left|A_{Q}\right|$ are even for each quad $Q$ in $S$ containing $a$. Since $A_{Q_{3}} \subseteq\{10\}$ and $\left|A_{Q_{3}}\right|$ is even, $10 \notin A_{Q_{3}}$ and so, $10 \notin B^{\prime}$. This argument with $Q_{3}$ replaced by $Q_{4}$ shows that $14 \notin B^{\prime}$. Since $A_{Q_{2}} \subseteq\{6,7,8\}$ and $\left|A_{Q_{2}}\right|$ is even, $j \notin B^{\prime}$ for some $j \in\{6,7,8\}$. Since $\left|B^{\prime}\right| \geq 3$ (Lemma 5.2(i)), $k \in B^{\prime}$ for some $k \in\{2,3,4,5\}$. Then, $A_{j, k}=\{k\}$, contradicting that $\left|A_{j, k}\right|$ is even.

\subsubsection{The near hexagon (iv)}

Let $Q_{1}, \cdots, Q_{6}$ be the six big quads in $S$ containing the point $a$. Any two of these big quads meet in a line through $a$ and any three of them meet only at $\{a\}$. Let

$$
\begin{aligned}
& Q_{1} \cap A=\left\{x_{1}, x_{2}, x_{3}, x_{4}, x_{5}\right\}, \\
& Q_{2} \cap A=\left\{x_{1}, x_{6}, x_{7}, x_{8}, x_{9}\right\}, \\
& Q_{3} \cap A=\left\{x_{2}, x_{6}, x_{10}, x_{11}, x_{12}\right\}, \\
& Q_{4} \cap A=\left\{x_{3}, x_{7}, x_{10}, x_{13}, x_{14}\right\}, \\
& Q_{5} \cap A=\left\{x_{4}, x_{8}, x_{11}, x_{13}, x_{15}\right\}, \\
& Q_{6} \cap A=\left\{x_{5}, x_{9}, x_{12}, x_{14}, x_{15}\right\} .
\end{aligned}
$$

Let $X=\left\{x_{1}, x_{2}, x_{3}, x_{4}, x_{6}, x_{7}, x_{8}, x_{10}, x_{11}\right\}$. Then $|X|=9$. We show that $X$ is a good subset of $A$. Assume otherwise. Since $Q_{6} \cap X$ is empty, $A_{Q_{6}}$ is empty and, by Lemma 5.2(ii), $\left|B^{\prime}\right|$ and $\left|A_{Q}\right|$ are even for every quad $Q$ in $S$ containing $a$. We first verify that for

$$
(i, j, k) \in\{(1,11,14),(1,12,13),(2,9,13),(3,6,15),(4,6,14),(5,6,13)\},
$$


$Q\left(x_{i}, x_{j}\right)$ is of type $(2,2)$ and $Q\left(x_{i}, x_{j}\right) \cap A=\left\{x_{i}, x_{j}, x_{k}\right\}$. Since $A_{1,12} \subseteq\{1\}$ and $\left|A_{1,12}\right|$ is even, it follows that $1 \notin B^{\prime}$. Similarly, considering $A_{2,9}$ and $A_{5,6}$, we conclude that $2 \notin B^{\prime}$ and $6 \notin B^{\prime}$. Since $6 \notin B^{\prime}$, considering $A_{3,6}$ and $A_{4,6}$, we conclude that $3 \notin B^{\prime}$ and $4 \notin B^{\prime}$. Since $\left|B^{\prime}\right| \geq 3$ is even, it follows that $B^{\prime}=\{7,8,10,11\}$ and so $A_{1,11}=\{11\}$, contradicting that $\left|A_{1,11}\right|$ is even.

\subsubsection{The near hexagon $(v)$}

Let $Q_{1}, Q_{2}, Q_{3}$ be the three big quads in $S$ containing $a$. Their intersection is $\{a\}$ and any two of these big quads meet in a line through $a$. We may assume that

$$
\begin{aligned}
& Q_{1} \cap A=\left\{x_{1}, x_{2}, x_{3}, x_{4}, x_{5}\right\}, \\
& Q_{2} \cap A=\left\{x_{1}, x_{6}, x_{7}, x_{8}, x_{9}\right\}, \\
& Q_{3} \cap A=\left\{x_{2}, x_{6}, x_{10}, x_{11}, x_{12}\right\} .
\end{aligned}
$$

Let $X=\left\{x_{1}, x_{2}, x_{3}, x_{4}, x_{6}, x_{7}, x_{8}, x_{10}, x_{11}\right\}$. Then $|X|=9$. We show that $X$ is a good subset of $A$. Assume otherwise. We note that the quads $Q\left(x_{r}, x_{k}\right)$ are of type $(2,2)$ in the following cases:

$$
r=1 \text { and } k \in\{10,11,12\} ; r=2 \text { and } k \in\{7,8,9\} ; r=6 \text { and } k \in\{3,4,5\} \text {. }
$$

Now, $A_{r, s} \subseteq\{r\}$ for $(r, s) \in\{(1,12),(2,9),(6,5)\}$ because $x_{s} \notin X$. Considering $A_{1,12}$, we conclude that $10,11 \notin B^{\prime}$ in view of the following: $A_{1,12} \subseteq\{1\}, A_{1, k} \subseteq$ $\{1, k\}$ for $k \in\{10,11\}$ and the parity of $\left|B^{\prime}\right|$ and $\left|A_{1, j}\right|$ are the same for all $j \neq 1$. Similarly, considering $A_{2,9}$ (respectively, $A_{6,5}$ ) we conclude that $7,8 \notin B^{\prime}$ (respectively, $3,4 \notin B^{\prime}$ ). Since $\left|B^{\prime}\right| \geq 3$, it follows that $B^{\prime}=\{1,2,6\}$. But $A_{5,9}$ is empty because $\left\{x_{5}, x_{9}, x_{12}\right\} \cap X$ and $\{10,11\} \cap B^{\prime}$ are empty. So $\left|B^{\prime}\right|$ is even (Lemma 5.2(ii)), a contradiction.

\subsection{The near hexagon $(v i)$}

We consider this case separately because the technique of the previous subsection only yields $|R| \geq 2^{17}$ in this case.

Let $S=(P, L)$ be a slim dense near hexagon and $Y$ be a proper subspace of $S$ isomorphic to the near hexagon (vii). Big quads in $Y$ (as well as in $S$ ) are of type $(2,4)$. There are three pair-wise disjoint big quads in $Y$ and any two of them generate $Y$. Fix two disjoint big quads $Q_{1}$ and $Q_{2}$ in $Y$. Let $(R, \psi)$ be a non-abelian representation of $S$. Set $M=\langle\psi(Y)\rangle$ and $M_{i}=M_{Q_{i}}$ for $i=1,2$. Then $\left|M_{i}\right|=2^{6}$ (Proposition 3.2(iii)), $M_{i} \cap Z(R)=\{1\}$ (Proposition 4.7), $M_{1} \cap M_{2}=\{1\}$ (Proposition 4.8). Since $Y$ contains pairs of points at distance 3, $(M, \psi)$ is a non-abelian representation of $Y$ (see Proposition 4.1 $(i)$ ). So, $M=2_{+}^{1+12}$ with $M=M_{1} M_{2} R^{\prime}$ (Theorem 1.6 for the near hexagon (vii)). Also, $R=M \circ N$, a central product of $M$ and $N=C_{R}(M)$.

Let $\{i, j\}=\{1,2\}$. For $x \in P \backslash Y$, we denote by $x^{j}$ the unique point in $Q_{j}$ at distance 1 from $x$. For $y \in Q_{i}$, let $z_{y}$ denote the unique point in $Q_{j}$ at distance 1 from $y$. For each $x \in P \backslash Y$, we can write $r_{x}=m_{1}^{x} m_{2}^{x} n_{x}$ for some $m_{1}^{x} \in M_{1}, m_{2}^{x} \in M_{2}$ and $n_{x} \in N$.

Proposition 5.3 For $x \in P \backslash Y, m_{i}^{x}=r_{z_{x} j}$. 
Proof Let $H_{j}=\left\langle r_{w}: w \in Q_{j} \cap x^{j \perp}\right\rangle \leq M_{j}$. Then $H_{j}$ is a maximal subgroup of $M_{j}$ ([10], 4.2.4, p. 68) and $r_{x} \in C_{R}\left(H_{1}\right) \cap C_{R}\left(H_{2}\right)$. For all $h \in H_{j}$,

$$
\left[m_{i}^{x}, h\right]=\left[m_{1}^{x} m_{2}^{x} n_{x}, h\right]=\left[r_{x}, h\right]=1 .
$$

So $m_{i}^{x} \in C_{M_{i}}\left(H_{j}\right)$. Note that $C_{M_{i}}\left(H_{j}\right)=\left\langle r_{z_{x} j}\right\rangle$, a subgroup of order 2. If $m_{i}^{x}=1$, then $r_{x}=m_{j}^{x} n_{x}$ commutes with every element of $M_{j}$. In particular, $\left[r_{x}, r_{y}\right]=1$ for every $y \in Q_{j} \cap \Gamma_{3}(x)$, a contradiction to Theorem 4.1(i). So $m_{i}^{x}=r_{z_{x} j}$.

Propositions 5.3 implies that $n_{x}$ is uniquely determined as $n_{x}=r_{x}\left(m_{1}^{x} m_{2}^{x}\right)^{-1}$.

Proposition 5.4 For $x \in P \backslash Y, n_{x}$ is an involution and $n_{x} \notin Z(R)$. In particular, $r_{x} \notin M$.

Proof By Proposition 2.3, $d\left(z_{x^{1}}, z_{x^{2}}\right)=2$. So $\left[m_{1}^{x}, m_{2}^{x}\right]=\left[r_{z_{x^{2}}}, r_{z_{x 1}}\right]=1$ (Proposition 5.3). Now, $r_{x}^{2}=1$ implies $n_{x}^{2}=1$. We show that $n_{x} \neq 1$ and $n_{x} \notin Z(R)$. The quad $Q=Q\left(x^{1}, x^{2}\right)$ in $S$ is of type $(2,2)$ or $(2,4)$ because $x^{1}$ and $x^{2}$ have at least three common neighbours $x, z_{x^{1}}$ and $z_{x^{2}}$. Let $U$ be the (2,2)-GQ in $Q$ generated by $\left\{x^{1}, x^{2}, x, z_{x^{1}}, z_{x^{2}}\right\}$. If $Q$ is of type $(2,4)$, then $\langle\psi(U)\rangle$ is of order $2^{5}$ (Corollary 3.4). If $Q$ is of type (2,2), then $U=Q$ is ovoidal because it is not a big quad. So $\langle\psi(U)\rangle$ is of order $2^{5}$ (Proposition 4.9). Therefore, $r_{a} r_{b} r_{c} \neq 1$ for every complete 3 arc $\{a, b, c\}$ of $U$ (Proposition 3.5). In particular, $n_{x}=r_{x} r_{z_{x 1}} r_{z_{x^{2}}} \neq 1$ for the complete 3 -arc $\left\{x, z_{x^{1}}, z_{x^{2}}\right\}$ of $U$. Now, applying Proposition 4.7 (respectively, Proposition 4.9) when $Q$ is of type $(2,4)$ (respectively, of type $(2,2)$ ), we conclude that $n_{x} \notin Z(R)$.

Proposition 5.5 Let $Q$ be a big quad in $S$ disjoint from $Y$ and $x, y \in Q$. Then:

(i) $\left[n_{x}, n_{y}\right]=1$ if and only if $x=y$ or $x \sim y$;

(ii) There is a unique line $l_{x}=\{x, y, x * y\}$ in $Q$ containing $x$ such that $n_{x * y}=n_{x} n_{y}$. For any other line $l=\{x, z, x * z\}$ in $Q, n_{x * z}=n_{x} n_{z} \theta$.

Proof $(i)$ Let $x \sim y$. By Propositions 2.5 and 5.3, $\left[m_{2}^{x}, m_{1}^{y}\right]=\left[m_{1}^{x}, m_{2}^{y}\right]=1$ or $\theta$. Then $\left[n_{x}, n_{y}\right]=\left[m_{1}^{x} m_{2}^{x} n_{x}, m_{1}^{y} m_{2}^{y} n_{y}\right]=\left[r_{x}, r_{y}\right]=1$.

Now, assume that $x \nsim y$. By Propositions 2.6 and 5.3, $\left\{\left[m_{1}^{x}, m_{2}^{y}\right],\left[m_{2}^{x}, m_{1}^{y}\right]\right\}=$ $\{1, \theta\}$. Since $\left[r_{x}, r_{y}\right]=1$, it follows that $\left[n_{x}, n_{y}\right]=\theta \neq 1$.

(ii) Let $x \in Q$ and $l_{x}$ be the line in $Q$ containing $x$ which corresponds to the line $x^{j} z_{x^{i}}$ in $Q_{j}$. This is possible by Lemma 2.2. For $u, v \in l_{x}, d\left(z_{u^{j}}, z_{v^{i}}\right) \leq 2$ (Proposition 2.5). So $\left[m_{i}^{u}, m_{j}^{v}\right]=1$. Then $r_{u * v}=\left(m_{1}^{u} m_{1}^{v}\right)\left(m_{2}^{u} m_{2}^{v}\right)\left(n_{u} n_{v}\right)$. So $n_{u * v}=$ $n_{u} n_{v}$. Let $l$ be a line $\left(\neq l_{x}\right)$ in $Q$ containing $x$. For $y \neq w$ in $l,\left[m_{2}^{y}, m_{1}^{w}\right]=\theta$ because $d\left(z_{y^{1}}, z_{w^{2}}\right)=3$ (Proposition 2.5). Then $r_{y * w}=\left(m_{1}^{y} m_{2}^{y} n_{y}\right)\left(m_{1}^{w} m_{2}^{w} n_{w}\right)=$ $\left(m_{1}^{y} m_{1}^{w}\right)\left(m_{2}^{y} m_{2}^{w}\right) n_{y} n_{w} \theta$ and so $n_{y * w}=n_{y} n_{w} \theta$.

Corollary 5.6 Let $Q$ be as in Proposition 5.5 and $I_{2}(N)$ be the set of involutions in $N$. Define $\delta$ from $Q$ to $I_{2}(N)$ by $\delta(x)=n_{x}, x \in Q$. Then

(i) $[\delta(x), \delta(y)]=1$ if and only if $x=y$ or $x \sim y$.

(ii) $\delta$ is one-to-one. 
(iii) There exists a spread $T$ in $Q$ such that for $x, y \in Q$ with $x \sim y$,

$$
\delta(x * y)=\left\{\begin{array}{ll}
\delta(x) \delta(y) & \text { if } x y \in T \\
\delta(x) \delta(y) \theta & \text { if } x y \notin T
\end{array} .\right.
$$

Proof $($ i) and (iii) follow from Proposition 5.5. We now prove (ii). Let $\delta(x)=$ $\delta(y)$ for $x, y \in Q$. By $(i), x=y$ or $x \sim y$. If $x \sim y$, then $r_{x * y}=r_{x} r_{y}=$ $\left(m_{1}^{x} m_{1}^{y}\right)\left(m_{2}^{x} m_{2}^{y}\right) \alpha \in M$, where $\alpha=\left[m_{2}^{x}, m_{1}^{y}\right] \in R^{\prime}$. But this is not possible as $x * y \notin Y$ (Proposition 5.4). So $x=y$.

Now, let $S=(P, L)$ be the near hexagon $(v i)$. Then big quads in $S$ are of type $(2,4)$. We refer to $([1]$, p. 363) for the description of the corresponding Fischer space on the set of 18 big quads in $S$. This set partitions into two families $F_{1}$ and $F_{2}$ of size 9 each such that each $F_{i}$ defines a partition of the point set $P$ of $S$. Let $U_{i}, i=1,2$, be the linear space whose point set is $F_{i}$. If $Q_{1}$ and $Q_{2}$ are two distinct points of $U_{i}$, then the line containing them is $\left\{Q_{1}, Q_{2}, Q_{1} * Q_{2}\right\}$, where $Q_{1} * Q_{2}$ is defined as in Lemma 2.2. Then $U_{i}$ is an affine plane of order 3 .

Consider the family $F_{1}$. Fix a line $\left\{Q_{1}, Q_{2}, Q_{1} * Q_{2}\right\}$ in $U_{1}$ and set $Y=Q_{1} \cup$ $Q_{2} \cup Q_{1} * Q_{2}$. Then $Y$ is a subspace of $S$ isomorphic to the near hexagon (vii). Fix a big quad $Q$ in $U_{1}$ disjoint from $Y$. Let the subgroups $M$ and $N$ of $R$ be as in the beginning of this subsection. Then $|N| \leq 2^{7}$ because $|R| \leq 2^{1+\operatorname{dim} V(S)}=2^{19}$. We show that $N=2_{-}^{1+6}$. This would prove Theorem 1.6 in this case.

Let $\left\{a_{1}, a_{2}, b_{1}, b_{2}\right\}$ be a quadrangle in $Q$, where $a_{1} \nsim a_{2}$ and $b_{1} \nsim b_{2}$. Let $\delta$ be as in Corollary 5.6. The subgroup $\left\langle\delta\left(a_{1}\right), \delta\left(a_{2}\right), \delta\left(b_{1}\right), \delta\left(b_{2}\right)\right\rangle$ of $R$ is isomorphic to $H=$ $\left\langle\delta\left(a_{1}\right), \delta\left(a_{2}\right)\right\rangle \circ\left\langle\delta\left(b_{1}\right), \delta\left(b_{2}\right)\right\rangle$. We write $N=H \circ K$ where $K=C_{N}(H)$. Then $|K| \leq$ $2^{3}$. There are three more neighbours, say $w_{1}, w_{2}, w_{3}$, of $a_{1}$ and $a_{2}$ in $Q$ different from $b_{1}$ and $b_{2}$. We can write

$$
\delta\left(w_{i}\right)=\delta\left(a_{1}\right)^{i_{1}} \delta\left(a_{2}\right)^{i_{2}} \delta\left(b_{1}\right)^{j_{1}} \delta\left(b_{2}\right)^{j_{2}} k_{i}
$$

for some $k_{i} \in K$, where $i_{1}, i_{2}, j_{1}, j_{2} \in\{0,1\}$. By Corollary 5.6(i), $\left[\delta\left(w_{i}\right), \delta\left(a_{r}\right)\right]=1$ and $\left[\delta\left(w_{i}\right), \delta\left(b_{r}\right)\right] \neq 1$ for $r=1,2$. This implies that $i_{1}=i_{2}=0$ and $j_{1}=j_{2}=1$. So $\delta\left(w_{i}\right)=\delta\left(b_{1}\right) \delta\left(b_{2}\right) k_{i}$. In particular, $k_{i}$ is of order 4 . Since $\left[\delta\left(w_{i}\right), \delta\left(w_{j}\right] \neq 1\right.$ for $i \neq j$, it follows that $\left[k_{i}, k_{j}\right] \neq 1$. Thus, $K$ is non-abelian of order 8 and $k_{1}, k_{2}, k_{3}$ are three pair-wise distinct elements of order 4 in $K$. So $K$ is isomorphic to $Q_{8}$ and $N=2_{-}^{1+6}$.

\section{References}

1. Brouwer, A.E., Cohen, A.M., Hall, J.I., Wilbrink, H.A.: Near polygons and Fischer spaces. Geom. Dedicata 49(3), 349-368 (1994)

2. Brouwer, A.E., Wilbrink, H.A.: The structure of near polygons with quads. Geom. Dedicata 14(2), 145-176 (1983)

3. Cameron, P.J.: Projective and polar spaces. Available from http://www.maths.qmul.ac.uk/ pjc/

4. De Bruyn, B.: Near Polygons. Frontiers in Mathematics. Birkhäuser, Basel (2006)

5. De Bruyn, B., Vandecasteele, P.: Near polygons with a nice chain of sub-near polygons. J. Combin. Theory Ser. A 108(2), 297-311 (2004) 
6. De Bruyn, B., Vandecasteele, P.: The classification of the slim dense near octagons. European J. Combin. 28(1), 410-428 (2007)

7. Doerk, K., Hawkes, T.: Finite Soluble Groups. de Gruyter Expositions in Mathematics, vol. 4. Walter de Gruyter \& Co., Berlin (1992)

8. Ivanov, A.A.: Non-abelian representations of geometries. Groups and combinatorics-in memory of Michio Suzuki. Adv. Stud. Pure Math. 32, 301-314 (2001) Math. Soc. Japan, Tokyo

9. Ivanov, A.A., Pasechnik, D.V., Shpectorov, S.V.: Non-abelian representations of some sporadic geometries. J. Algebra 181(2), 523-557 (1996)

10. Payne, S.E., Thas, J.A.: Finite Generalized Quadrangles. Research Notes in Mathematics, vol. 110. Pitman (Advanced Publishing Program), Boston (1984)

11. Ronan, M.A.: Embeddings and hyperplanes of discrete geometries. European J. Combin. 8(2), 179185 (1987)

12. Sahoo, B.K., Sastry, N.S.N.: A characterization of finite symplectic polar spaces of odd prime order. J. Combin. Theory Ser. A 114, 52-64 (2007)

13. Shult, E., Yanushka, A.: Near n-gons and line systems. Geom. Dedicata 9(1), 1-72 (1980) 